\title{
Direct observation of protonation states in a PLP-dependent enzyme by neutron crystallography
}

Pyridoxal-5'-phosphate (PLP, vitamin B6) is one of the most ubiquitous co-factors in biological systems. PLP-dependent enzymes are essential proteins that catalyze a diverse number of reactions: transamination, racemization, phosphorylation, decarboxylation, aldol cleavage, elimination, and replacement. At least five different fold types comprise the PLP-dependent families of enzymes, each catalyzing a specific type of chemistry. For several decades, PLPdependent enzyme research has devolved the hypothesis that the electronics/electrophilicity of PLP are modulated to optimize the desired chemical reaction in each family of enzymes. One way to modulate the electronics/electrophilicity is to invoke different protonation states of PLP. The active site local environment can promote the different protonation profiles of PLP during the catalytic cycle. Here, we report the first neutron crystal structure of a PLP dependent enzyme, aspartate aminotransferase (AAT). Neutron protein crystallography is one of the most powerful modern techniques to study the positions of hydrogen/deuterium atoms in a macromolecule. The AAT crystal was soaked with $\alpha$-methyl-DL-aspartic acid (substrate derivative) to capture AAT in both the ground (internal aldimine) and substrate bound (external aldimine) states. Interestingly, the neutron structure of AAT shows that PLP and active site residues have different protonation/deuteration states in the ground vs. substrate bound states. In the efforts to validate our hypothesis, an interdisciplinary approach using neutron/X-ray diffraction and molecular modeling has be implemented to determine the specific chemical mechanisms.

$\begin{array}{ll}\text { Steven Dajnowicz } & \text { University of Toledo } \\ \text { Matthew Blakeley } & \text { Institut Max Von Laue - Paul Langevin } \\ \text { David Keen } & \text { Rutherford Appleton Laboratory } \\ \text { AndreyKovalevsky } & \text { Oak Ridge National Laboratory } \\ \text { Timothy Mueser } & \text { University of Toledo }\end{array}$

